\title{
Do Lifestyle Factors Including Smoking, Alcohol, and Exercise Impact Your Risk of Developing Kidney Stone Disease? Outcomes of a Systematic Review
}

\author{
Patrick Jones, MRCS, ${ }^{1}$ Sadaf Karim Sulaiman, MRCS, ${ }^{1}$ Kithmini N. Gamage, MBChB, \\ Theodoras Tokas, MD, PhD, FEBU, ${ }^{2}$ Enakshee Jamnadass, MBChB, ${ }^{1}$ \\ and Bhaskar K. Somani, MRCS, DM, FEBU, FFSTEd, FRCS (Urol $)^{1}$
}

\begin{abstract}
Introduction: With a rise in the incidence of stone disease, more research is needed to understand the lifestyle factors associated with it. We evaluate available evidence for association of smoking, alcohol, and exercise with kidney stone disease (KSD).

Methods: A systematic literature search was conducted in CINAHL, EMBASE, Ovid Medline, Cochrane Library, Scopus, Clinicaltrials.gov, and Google Scholar. Different keywords were "smoking," "cigarette," "alcohol," "spirits," "exercise," "physical activity," "training," "kidney stone," "stone disease," "nephrolithiasis," "urolithiasis," "renal stone," and "urinary stone." The main outcome of interest was the role of smoking, alcohol, and exercise in the development of KSD.

Results: A total of 4921 articles were found on searching the databases, of which 14 met the criteria for inclusion in the final review. Studies assessed the following risk factors, physical activity $(n=6)$, alcohol $(n=6)$, and smoking $(n=9)$, and included 17,511 patients. The relationship of physical activity with KSD appears to be equivocal. In addition, only one study demonstrated a decreased risk of nephrolithiasis with alcohol consumption. On the contrary, four studies found a significant association between smoking and renal stone formation. Conclusions: While smoking is one behavioral factor that seems to have some association with KSD, no clear effect of alcohol and physical activity has been demonstrated. To avoid KSD, awareness of the possible detrimental role of smoking should be considered and patients should remain vigilant about the importance of hydration with physical activity. While this represents the most appropriate guidance from the evidence available, at present there remain insufficient data to truly reveal the relationship between these three factors and KSD.
\end{abstract}

Keywords: kidney stones, nephrolithiasis, renal stones, lifestyle, smoking, alcohol, exercise, prevention

\section{Introduction}

$\mathbf{T}$ HE LIFETIME PREVALENCE of kidney stone disease (KSD) is $14 \%$ and epidemiologic data have confirmed this is increasing. ${ }^{1,2}$ Furthermore, at least $50 \%$ of the patients will experience a stone recurrence within 10 years. ${ }^{3}$ The burden of KSD is far-reaching, both at an individual and society level. ${ }^{4,5}$ As well as the detrimental effects on a patient's quality of life, the economic sequelae are also extensive. The annual cost is estimated to exceed $\$ 4$ billion in the United States by 2030, notwithstanding the additional costs incurred by a patient for lost work. ${ }^{4}$ Geraghty and colleagues reported the average stone episode in the United Kingdom to be as high as $£ 2187$, with the cost of KSD being comparable with the combined cost of bladder and prostate cancer. ${ }^{5}$

While treatment methods have improved with regard to efficacy and safety, research and development are also required to continue understanding the etiology and preventative measures. ${ }^{6}$ The association between an array of dietary factors such as salt, animal protein, and urinary stone formation is now well recognized. ${ }^{7,8}$ Other lifestyle factors such as smoking, alcohol, and physical activity have also been incriminated to play a role in lithogenesis. ${ }^{9}$ However, evidence and recommendations remain underreported. Each of

\footnotetext{
${ }^{1}$ Department of Urology, University Hospital Southampton NHS Trust, Southampton, United Kingdom.

${ }^{2}$ Department of Urology and Andrology, General Hospital Hall in Tirol, Tirol, Austria.
} 
these represents a modifiable risk factor and further attention is therefore warranted. This is especially true given the prevalence of poor lifestyle behaviors and choices. Of note, in 2010 , it was reported that $28.1 \%$ of the population in China were smokers. ${ }^{10}$ The possible implications and the potential for remedial action are therefore immense. This review aims to evaluate the available evidence for association of lifestyle factors such as smoking, alcohol, and exercise with KSD, with the intention that clinical recommendations can be drawn to help prevent primary and recurrent stone disease.

\section{Materials and Methods}

\section{Evidence acquisition}

Inclusion criteria

1. Studies in English language looking at the of role of smoking, alcohol, and/or exercise in KSD.

2. Studies over the past two decades.

3. Adult patients.

4. Studies that clearly recorded separate intake of alcohol and smoking to allow for subsequent analysis and possible identification of independent risk factors.

\section{Exclusion criteria}

1. Animal and laboratory studies.

2. Review articles and gray literature.

3. Studies looking at the role of dietary factors or volume of water intake in KSD.

\section{Search strategy and study selection}

A systematic literature search was conducted according to the Cochrane specifications and Preferred Reporting Items for Systematic Reviews and Meta-analyses (PRISMA) protocol. ${ }^{11}$ The search strategy was devised and conducted to find relevant articles from bibliographic databases, including CINAHL, EMBASE, Ovid Medline, Cochrane Library, Scopus, Clinicaltrials.gov, and Google Scholar. The search was confined to all English-language articles between January 2000 and January 2020. Search terms included (but were not limited to): "smoking," "cigarette," "alcohol," "spirits," “exercise," "physical activity," “training," "kidney stone," "stone disease," "nephrolithiasis," "urolithiasis," "renal stone," and "urinary stone." Boolean operators (AND, OR) were used to augment the search. Two reviewers (S.S. and P.J.) identified all studies independently and any discrepancies were resolved by the senior author (B.K.S.). When additional information or clarification was needed, the primary authors of the studies were contacted directly. The main outcome of interest was the role of smoking, alcohol, and exercise in the development of KSD. Older articles, as well as animal and laboratory studies, and reviews related to the subject are described in a purely narrative manner and not included in the qualitative analysis.

\section{Analysis}

A narrative approach was adopted to analyze the data given its heterogeneous nature. It was determined by mutual consensus between the authors that it was not appropriate to carry out a formal meta-analysis using, for example, a global estimate effect. The wide range of cutoff values would also likely impact this. The Newcastle/Ottawa Scale (NOS) was implemented for quality appraisal. ${ }^{12}$

\section{Results}

A total of 4921 articles were found on searching the databases, of which 140 abstracts were reviewed, and 14 articles met the criteria for inclusion in the final review (Fig. 1). Of

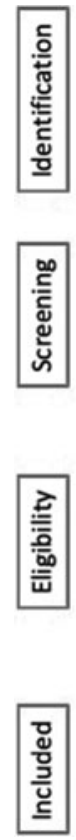

Records identified through database searching and removing duplicates $(n=4921)$

Articles excluded after screening of title ( $n=4781$ )

Potential articles for evaluation of abstract ( $n=140$ )

Articles excluded after screening of abstract ( $n=106$ )

Full-text articles assessed fo eligibility $(n=34)$

Full-text articles excluded $(n=20)$

Included articles $(n=14)$

FIG. 1. PRISMA flowchart of the included studies. PRISMA = Preferred Reporting Items for Systematic Reviews and Meta-Analyses. 
them, 11 were case/control and the remainder were cohort studies. All of these applied a questionnaire as an assessment tool. In total, data from 17,511 patients were included. Studies assessed the following risk factors: physical activity $(n=6)$, alcohol $(n=6)$, and smoking $(n=9)$ (Table 1).

\section{Physical activity}

Three studies reported no effect of physical activity on KSD risk. ${ }^{13-15}$ In contrast, two groups reported a statistically significant protective role of exercise. ${ }^{16,17}$ Sorensen and colleagues reported a reduction risk of stone formation associated with physical activity of women and this was independent of intensity of activity, caloric intake, and body mass index. ${ }^{16}$ More specifically, compared with inactive women, the risk of incident stones decreased by $16 \%$ in women with the lowest physical activity level (adjusted hazard ratio [aHR] 0.84; 95\% confidence interval [95\% CI] 0.74-0.97). By increasing activity, the risk of incident stones declined further reaching a decrease of $\sim 31 \%$ (aHR 0.69 ; 95\% CI $0.60-0.79) .{ }^{16}$ Furthermore, Zhuo et al. showed that the duration of physical activity was an independent risk factor significantly associated with the occurrence of KSD (OR 0.840; 95\% CI 0.808-0.973). ${ }^{17}$ In contrast, Zhao and colleagues concluded that daily exercise greater than 30 minutes in duration could significantly increase the risk of KSD (OR 2.1; 95\% CI 1.3-3.5). ${ }^{18}$

Ferraro and colleagues suggested that the reason their nonrandomized study did not find any effect of exercise on KSD was due to unmeasured and/or unknown variables. ${ }^{13}$ Maric and colleagues were also unable to add any reasoning for the lack of association. ${ }^{15}$ The former study was limited by including a sample of nearly all Caucasian patients, no patients younger than 40 years, and no breakdown of stone composition. Five out of the six studies therefore showed no detrimental effect of exercise on risk of KSD, while one study found it detrimental but only in those who completed more than 30 minutes of physical activity. It may be therefore that overexercising and dehydration are risk factors for KSD.

\section{Alcohol}

Six studies determined no significant effect of alcohol intake on urinary stone disease. ${ }^{18-23}$ However, in a classic twin study analysis by Goldfarb et al., it was found that alcohol consumption in the past 2 weeks was associated with a decreased risk of nephrolithiasis $(\mathrm{OR}=2.7 ; 95 \% \mathrm{CI} 1.0-7.7){ }^{24}$ Nonetheless, the same group found no significant association for lifetime consumption of at least 20 alcoholic beverages, daily intake of wine or hard liquor in the past 2 weeks, or alcohol intake in the past month. Finally, this study reported a hereditary link and a protective effect of coffee and perhaps tea, fruits, and vegetables.

\section{Smoking}

Five of the studies that investigated the effect of smoking on KSD showed no positive correlation. ${ }^{15,18,19,21,25}$ However, the remaining four studies that assessed this did find a significant association. ${ }^{14,22,23,26}$ More specifically, Hamano and colleagues demonstrated an increased significance of smoking risk for KSD in a multivariate logistic regression analysis (OR 4.29; 95\% CI 2.68-6.86; $p<0.0001) .{ }^{23}$ In ad- dition, Liu and colleagues defined cigarette smoking as an independent risk factor (OR 1.66; 95\% CI 1.11-2.50; $p=0.014$ ) for calcium urolithiasis development. ${ }^{22}$ Moreover, Tamadon and colleagues recorded a significant increase of nephrolithiasis risk with smoking (OR 2.06; 95\% CI 1.06$4.01 ; p=0.034)$. However, no significant difference in the number of cigarettes smoked $(p=0.830)$ and years of smoking $(p=0.536)$ between subjects with and without stones $(p=0.536)$ was found. ${ }^{26}$ Finally, another survey study by Soueidan and colleagues showed that KSD patients had higher rates of smoking $(7 \%$ vs $21 \%, p=0.02)$ and were 8.5 times more likely (95\% CI 2.2-32.2) to be current smokers. ${ }^{14}$

\section{Quality appraisal of studies}

Overall, there was a good quality of evidence of included studies, as the scientific rationale and study objectives were clearly documented, and the selection and definitions for control groups were well done. Most case/control studies adequately drew the nonexposed cohort from the same community as the exposed cohort, rather than from a different source or not providing any description of their derivation at all. The average appraisal score using the NOS was 5.6/9 (range 5-7) (Table 2). Areas of weakness across studies included the method of outcome assessment. Few studies implemented an independent blind assessment. Instead, they used either self-report or provided no description.

\section{Discussion \\ Key findings}

While the European Association of Urology (EAU) acknowledges lifestyle factors in the development of KSD, this is not expanded upon. ${ }^{27}$ Given the known relationship between coronary heart disease (CHD) and KSD, in theory, it would be anticipated that risk factors for CHD could also contribute to KSD at least indirectly. ${ }^{13}$ Similarly, the association between metabolic syndrome (which includes obesity, hypertension, high triglyceride levels, and diabetes) and KSD is well established, ${ }^{8}$ while lifestyle factors such as physical activity, alcohol, and smoking, all, in part, directly or indirectly cause metabolic syndrome.

\section{Link to activity}

There have been few studies investigating the relationship between physical activity and KSD. Given the widely known cardiovascular benefits of regular exercise, in theory, it should serve a protective role as it also decreases the risk of metabolic syndrome and improves hypertension. ${ }^{28}$ While the findings by Zhao and colleagues at first seem contradictory to this logic, a closer review reveals why this is likely the case. ${ }^{18}$ Their study would infer a patient's overexercising and perhaps not hydrating sufficiently. The urine would, therefore, be concentrated and this could potentially increase the risk of crystallization and stone formation. This is supported by earlier studies that found marathon runners to be at greater risk of KSD. ${ }^{29}$ Given that prolonged immobility and the resultant bone mineral loss are associated with $\mathrm{KSD},{ }^{30}$ it serves that moderate exercise should be recommended for KSD patients. 


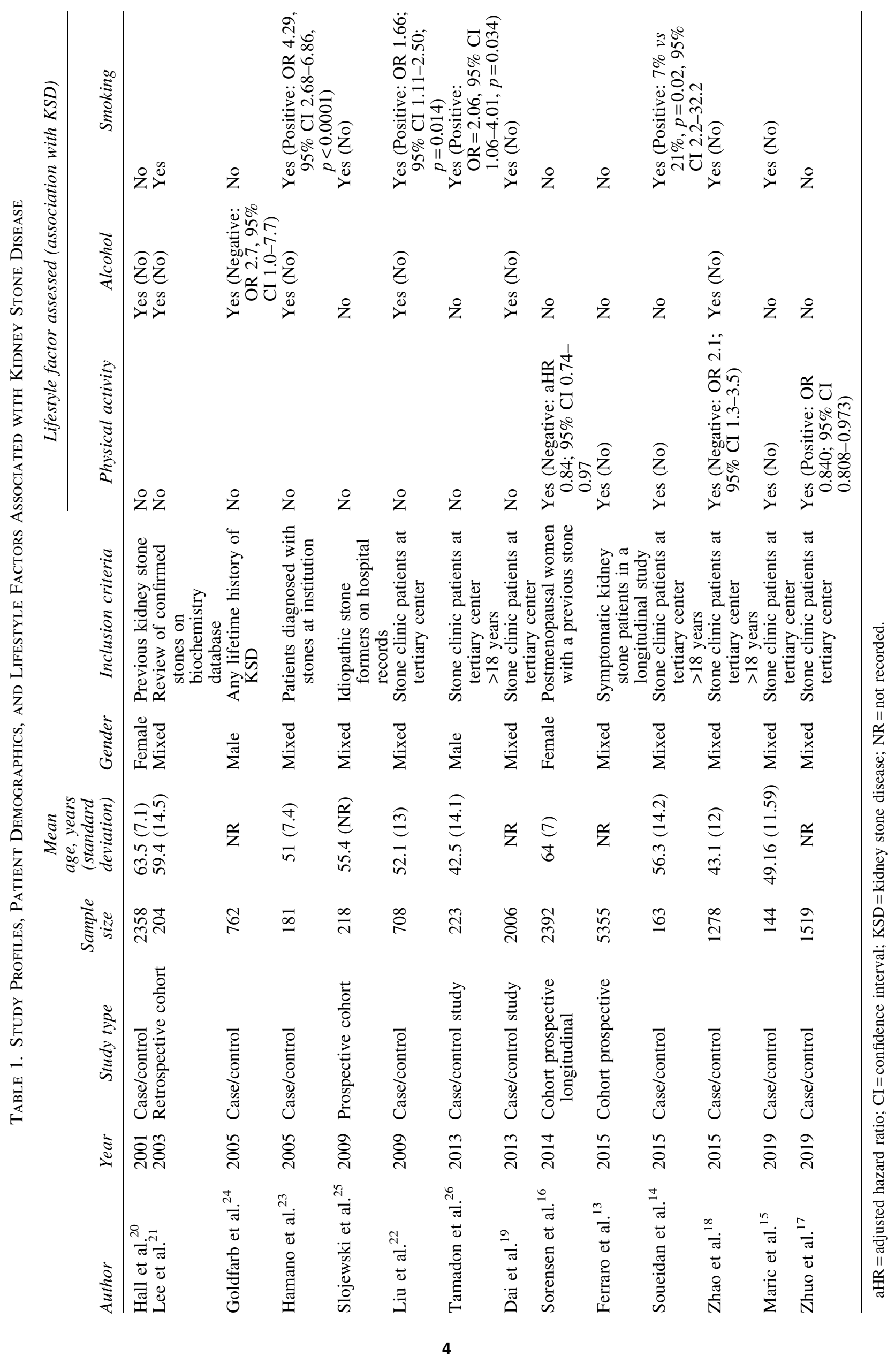


Table 2. Lifestyle Effect of Physical Activity, Smoking, and Alcohol and Quality Appraisal of the Studies

\begin{tabular}{|c|c|c|c|c|c|c|}
\hline \multirow[b]{2}{*}{ Author } & \multirow{2}{*}{$\begin{array}{l}\text { Quality } \\
\text { Appraisal } \\
\text { score } \\
\text { (NOS: 0-9) }\end{array}$} & \multirow[b]{2}{*}{ Assessment } & \multicolumn{3}{|c|}{ Lifestyle factor effect } & \multirow[b]{2}{*}{ Additional comments } \\
\hline & & & $\begin{array}{l}\text { Physical } \\
\text { activity }\end{array}$ & Alcohol & Smoking & \\
\hline Hall et al. ${ }^{20}$ & 5 & Questionnaire & N/A & Nil & N/A & $\begin{array}{l}\text { No association found between alcohol } \\
\text { and KSD }\end{array}$ \\
\hline Lee et $\mathrm{al}^{21}$ & 5 & Questionnaire & N/A & Nil & Nil & $\begin{array}{l}\text { No association found between } \\
\text { smoking/alcohol and KSD }\end{array}$ \\
\hline Goldfarb et al. ${ }^{24}$ & 5 & Questionnaire & N/A & Decrease $^{\mathrm{a}}$ & N/A & $\begin{array}{l}\text { Nondrinkers more likely to report KSD } \\
(\mathrm{OR}=2.7,95 \% \text { CI } 1.0-7.7)\end{array}$ \\
\hline Hamano et al. ${ }^{23}$ & 6 & Questionnaire & N/A & N/A & Increase $^{\mathrm{a}}$ & $\begin{array}{l}\text { (70.2\% vs } 25.1 \%, p<0.0001 \\
\text { Risk of KSD increased by } 4.3\end{array}$ \\
\hline Slojewski et al. ${ }^{25}$ & 5 & Questionnaire & N/A & N/A & Nil & $\begin{array}{l}\text { No association found between smoking } \\
\text { and KSD }\end{array}$ \\
\hline Liu et al. ${ }^{22}$ & 5 & Questionnaire & N/A & Nil & Increase $^{\mathrm{a}}$ & $\begin{array}{l}\text { Smoking is an independent risk factor } \\
\text { for KSD but alcohol is not }\end{array}$ \\
\hline Tamadon et al. $^{26}$ & 6 & Questionnaire & N/A & N/A & Increase $^{\mathrm{a}}$ & $\begin{array}{l}\text { Risk of stone formation doubled } \\
\text { No significant difference between } \\
\text { numbers smoked }\end{array}$ \\
\hline Dai et al. ${ }^{19}$ & 7 & Questionnaire & N/A & Nil & Nil & $\begin{array}{l}\text { No association found between } \\
\text { smoking/alcohol and KSD }\end{array}$ \\
\hline Sorensen et al. ${ }^{16}$ & 6 & Questionnaire & Decrease $^{a}$ & N/A & N/A & $\begin{array}{l}\text { Reduced risk of stone formation } \\
\text { independent of calorie intake and } \\
\text { BMI }\end{array}$ \\
\hline Ferraro et al. ${ }^{13}$ & 7 & Questionnaire & Nil & N/A & N/A & $\begin{array}{l}\text { No association between activity and } \\
\text { KSD }\end{array}$ \\
\hline Soueidan et al. ${ }^{14}$ & 6 & Questionnaire & Nil & N/A & Increase $^{\mathrm{a}}$ & $\begin{array}{l}\text { Recurrent stone former } 8.5 \text { times for } \\
\text { likely to be smoker }\end{array}$ \\
\hline Zhao et al. ${ }^{18}$ & 5 & Questionnaire & Increase $^{\mathrm{a}}$ & Nil & Nil & $\begin{array}{l}\text { Exercise }>30 \text { minutes increases risk of } \\
\text { KSD } \\
\text { No effect of smoking and alcohol on } \\
\text { KSD }\end{array}$ \\
\hline Maric et al. ${ }^{15}$ & 5 & Questionnaire & Nil & N/A & Nil & $\begin{array}{l}\text { Activity level and smoking had no } \\
\text { effect on risk of KSD }\end{array}$ \\
\hline Zhuo et al. ${ }^{17}$ & 5 & Questionnaire & Decrease $^{a}$ & N/A & N/A & $\begin{array}{l}\text { Physical activity protective on } \\
\text { multivariate logistic regression } \\
\text { analysis }\end{array}$ \\
\hline
\end{tabular}

${ }^{\mathrm{a}}$ Where changes are statistically significant $(p<0.05)$.

$\mathrm{BMI}=$ body mass index; NOS = Newcastle/Ottawa Scale.

\section{Link to alcohol}

While most of the clinical studies have not shown an increased risk of KSD development with increased alcohol intake, there exists disagreement as to whether alcohol consumption should induce stone formation or be protective. Siener found that subjects consuming alcohol excreted more calcium, which resulted in transient hypercalciuria that could lead to greater chance of calcium oxalate stone formation. ${ }^{31}$ Animal studies have shown that rats treated with ethanol develop crystal formation. ${ }^{32}$ Alcohol is also suggested to increase the production of uric acid and may contribute to uric acid stones. ${ }^{33}$ In addition, alcohol consumption can cause oxidative stress to renal tissue, which can lead to lithogenesis. However, red wine contains antioxidants, and this could offset this harm. ${ }^{34}$ Arguments for a protective role are largely derived from the dilution of metabolites in blood and urine. The known diuretic effect can increase urinary output, which is a protective factor and can reduce the risk of KSD. ${ }^{35}$ In general, there are a limited number of clinical studies investigating this topic, given that alcohol consumption is lower in certain countries for cultural or religious reasons.

\section{Link to smoking}

Smoking is postulated to increase the risk of KSD through a multitude of factors. ${ }^{23}$ One hypothesis is related to the increase in vasopressin levels, a strong antidiuretic, which can lead to poor urinary flow and a low urine output, which is further associated with greater risk of KSD. ${ }^{36}$ Moreover, increasing levels of urinary metallic elements such as strontium and mercury, which are present in higher levels in smokers, may be of significance, but their relevance is inconclusive at present. $^{22,25}$ It was also found that smoking decreases the level of urinary calcium excretion, which is proven to be a risk factor of KSD. ${ }^{36}$ Smoking also causes the release of reactive oxygen species and resultant oxidative stress on the kidneys. This renal injury can accelerate the onset of chronic kidney disease, which is an established risk factor for lithogenesis. Workers in certain construction 
industries who experience chronic exposure to cadmium are known to carry a higher risk of KSD. Cigarettes raise the plasma concentration of cadmium and this may also contribute to increased risk. ${ }^{37}$

\section{Strengths, limitations, and areas of future research}

This is the first systematic review to assess different lifestyle factors, including smoking, alcohol, and exercise, and their impact on developing KSD. It has been prepared according to the Cochrane guidelines and PRISMA checklist, summarizing available data on this topic. It gives an overview of the studies on the link of KSD with lifestyle factors, although none of them was randomized control trials. Furthermore, a lack of heterogeneity of included studies precluded a formal meta-analysis. We could not establish or compare the level of activity, pack-years of smoking, units, and duration of alcohol intake from the studies. The possible presence of many uncontrolled or unknown confounding variables was also likely to impede proving direct causation of smoking, alcohol, and exercise. As well as possible selection bias, we also acknowledge the publication bias and that the conclusions drawn are only as robust as the included studies. Furthermore, some studies included only male or female patients, which is a further limitation on the possible conclusions.

One of the additional problems that are highlighted among many of the epidemiologic studies is that behavioral habits such as smoking and alcohol consumption are often coupled, and it is difficult to carry out subgroup analyses to separate these as independent risk factors. The nature of the assessment method in all studies using questionnaires introduces recall bias. Of particular relevance to this review is the role of social desirability bias as patients may have unconsciously underreported their smoking or drinking habits as well as overestimated physical activity. Rzewnicki and colleagues reported that overestimating physical activity in questionnaire studies is common. ${ }^{38}$ Although no randomized studies exist, this would arguably be unethical to be done in the context of smoking and alcohol. An assessment of the association between warm weather, exercise, and hydration is perhaps also warranted. ${ }^{39}$ Further longitudinal multicentric studies are still needed, however, to give further insight into additional factors such as exercise intensity, hydration levels, pack-years of smoking, units of alcohol consumed, and quality of life of these patients. ${ }^{40}$ Future studies should also aim to investigate the relationship between the risk of stone formation and also the risk of stone recurrence.

\section{Conclusion}

While smoking is one behavioral factor that seems to have some association with KSD, no clear effect of alcohol has been demonstrated, and physical activity although helpful was negated by dehydration and hence increased the risk of KSD. To avoid KSD, awareness of the possible detrimental role of smoking should be considered and patients should remain vigilant about the importance of hydration with physical activity. While this represents the most appropriate guidance from the evidence available, at present there remain insufficient data to truly reveal the relationship between these three factors and KSD.

\section{Author Disclosure Statement}

No competing financial interests exist.

\section{Funding Information}

No funding was received for this article.

\section{References}

1. Rukin NJ, Siddiqui ZA, Chedgy ECP, Somani BK. Trends in upper tract stone disease in England: Evidence from the Hospital Episodes Statistics Database. Urol Int 2017;98: 391-396.

2. Turney BW, Reynard JM, Noble JG, Keoghane SR. Trends in urological stone disease. BJU Int 2012;109:1082-1087.

3. Knoll T. Epidemiology, pathogenesis, and pathophysiology of urolithiasis. Eur Urol Suppl 2010;9:802-806.

4. Antonelli JA, Maalouf NM, Pearle MS, Lotan Y. Use of the National Health and Nutrition Examination Survey to calculate the impact of obesity and diabetes on cost and prevalence of urolithiasis in 2030. Eur Urol 2014;66:724-729.

5. Geraghty RM, Cook P, Walker V, Somani BK. Evaluation of the economic burden of kidney stone disease in the UK: A retrospective cohort study with a mean follow-up of 19 years. BJU Int 2020;125:586-594.

6. Geraghty RM, Jones P, Somani BK. Worldwide trends of urinary stone disease treatment over the last two decades: A systematic review. J Endourol 2017;31:547-556.

7. Denburg MR, Jemielita TO, Tasian GE, Haynes K, Mucksavage P, Shults J, Copelovitch L. Assessing the risk of incident hypertension and chronic kidney disease after exposure to shock wave lithotripsy and ureteroscopy. Kidney Int 2016;89:185-192.

8. Wong Y, Cook P, Roderick P, Somani BK. Metabolic syndrome and kidney stone disease: A systematic review of literature. J Endourol 2016;30:246-253.

9. Anderson RA. A complementary approach to urolithiasis prevention. World J Urol 2002;20:294-301.

10. Li Q, Hsia J, Yang G. Prevalence of smoking in China in 2010. N Engl J Med 2011;364:2469-2470.

11. Moher D, Liberati A, Tetzlaff J, Altman DG. Preferred reporting items for systematic reviews and meta-analyses: The PRISMA statement. PLoS Med 2009;6:e1000097.

12. Stang A. Critical evaluation of the Newcastle-Ottawa scale for the assessment of the quality of nonrandomized studies in meta-analyses. Eur J Epidemiol 2010;25:603-605.

13. Ferraro PM, Curhan GC, Sorensen MD, Gambaro G, Taylor EN. Physical activity, energy intake and the risk of incident kidney stones. J Urol 2015;193:864-868.

14. Soueidan M, Bartlett SJ, Noureldin YA, Andersen RE, Andonian S. Leisure time physical activity, smoking and risk of recent symptomatic urolithiasis: Survey of stone clinic patients. Can Urol Assoc J 2015;9:257-262.

15. Maric I, Kizivat T, Smolic M, et al. Lifestyle risk factors and bone mass in recurrent stone-forming patients: A crosssectional study in 144 subjects. Acta Clin Croat 2019;58: 439-445.

16. Sorensen MD, Chi T, Shara NM, et al. Activity, energy intake, obesity, and the risk of incident kidney stones in postmenopausal women: A report from the Women's Health Initiative. J Am Soc Nephrol 2014;25:362-369.

17. Zhuo D, Li M, Cheng L, Zhang J, Huang H, Yao Y. A study of diet and lifestyle and the risk of urolithiasis in 1,519 patients in Southern China. Med Sci Monit 2019;25: 4217-4224. 
18. Zhao A, Dai M, Chen YJ, Chang HE, Liu AP, Wang PY. Risk factors associated with nephrolithiasis: A case-control study in China. Asia Pac J Public Health 2015;27:Np414-Np424.

19. Dai M, Zhao A, Liu A, You L, Wang P. Dietary factors and risk of kidney stone: A case-control study in southern China. J Ren Nutr 2013;23:e21-e28.

20. Hall WD, Pettinger M, Oberman A, Watts NB, Johnson KC, Paskett ED, Limacher MC, Hays J. Risk factors for kidney stones in older women in the southern United States. Am J Med Sci 2001;322:12-18.

21. Lee YH, Huang WC, Lu CM, Tsai JY, Huang JK. Stone recurrence predictive score (SRPS) for patients with calcium oxalate stones. J Urol 2003;170(Pt 1):404-407.

22. Liu CC, Huang SP, Wu WJ, Chou YH, Juo SH, Tsai LY, Huang $\mathrm{CH}$, Wu MT. The impact of cigarette smoking, alcohol drinking and betel quid chewing on the risk of calcium urolithiasis. Ann Epidemiol 2009;19:539-545.

23. Hamano S, Nakatsu H, Suzuki N, Tomioka S, Tanaka M, Murakami S. Kidney stone disease and risk factors for coronary heart disease. Int J Urol 2005;12):859-863.

24. Goldfarb DS, Fischer ME, Keich Y, Goldberg J. A twin study of genetic and dietary influences on nephrolithiasis: A report from the Vietnam Era Twin (VET) Registry. Kidney Int 2005;67):1053-1061.

25. Slojewski M, Czerny B, Safranow K, et al. Does smoking have any effect on urinary stone composition and the distribution of trace elements in urine and stones? Urol Res 2009;37:317-322.

26. Tamadon MR, Nassaji M, Ghorbani R. Cigarette smoking and nephrolitiasis in adult individuals. Nephrourol Mon 2013;5:702-705.

27. Turk C, Petrik A, Sarica K, Seitz C, Skolarikos A, Straub M, Knoll T. EAU Guidelines on Diagnosis and Conservative Management of Urolithiasis. Eur Urol 2016;69:468-474.

28. Meschi T, Nouvenne A, Borghi L. Lifestyle recommendations to reduce the risk of kidney stones. Urol Clin North Am 2011;38:313-320.

29. Irving RA, Noakes TD, Rodgers AL, Swartz L. Crystalluria in marathon runners. 1. Standard marathon-Males. Urol Res 1986;14:289-294.

30. Kohri K, Yasui T, Okada A. Space flight/bedrest immobilization and bone. Urolithiasis formation during space flight and long-term bed rest [Jpn]. Clin Calcium 2012;22:1821-1828.

31. Siener R. Impact of dietary habits on stone incidence. Urol Res 2006;34:131-133.

32. Kuo RL, Lingeman JE, Evan AP, Paterson RF, Parks JH, Bledsoe SB, Munch LC, Coe FL. Urine calcium and volume predict coverage of renal papilla by Randall's plaque. Kidney Int 2003;64:2150-2154.

33. Zechner O, Scheiber V. The role of affluence in recurrent stone formation. In: Smith LH, Robertson WG, Finlayson B, eds. Urolithiasis. Boston, MA: Springer, 1981, pp. 309-313.
34. Zecher M, Guichard C, Velasquez MJ, Figueroa G, Rodrigo R. Implications of oxidative stress in the pathophysiology of obstructive uropathy. Urol Res 2009;37:19-26.

35. Hirvonen T, Pietinen P, Virtanen M, Albanes D, Virtamo J. Nutrient intake and use of beverages and the risk of kidney stones among male smokers. Am J Epidemiol 1999;150: 187-194.

36. Sulaiman SK, Enakshee J, Traxer O, et al. Which type of water is recommended for patients with stone disease (hard or soft water, tap or bottled water): Evidence from a systematic review over the last 3 decades. Curr Urol Rep 2020; 21:6.

37. Mooser V, Burnier M, Nussberger J, Juillerat L, Waeber B, Brunner HR. Effects of smoking and physical exercise on platelet free cytosolic calcium in healthy normotensive volunteers. J Hypertens 1989;7:211-216.

38. Rzewnicki R, Vanden Auweele Y, De Bourdeaudhuij I. Addressing overreporting on the International Physical Activity Questionnaire (IPAQ) telephone survey with a population sample. Public Health Nutr 2003;6:299-305.

39. Geraghty RM, Proietti S, Traxer O, Archer M, Somani BK. Worldwide impact of warmer seasons on the incidence of renal colic and kidney stone disease: Evidence from a systematic review of literature. J Endourol 2017;31:729-735.

40. New F, Somani BK. A complete world literature review of quality of life (QOL) in patients with kidney stone disease (KSD). Curr Urol Rep 2016;17:88.

Address correspondence to: Bhaskar K. Somani, MRCS, DM, FEBU, FFSTEd, FRCS (Urol) Department of Urology University Hospital Southampton NHS Trust Southampton SO16 6YD United Kingdom

E-mail: bhaskarsomani@yahoo.com

$\quad$ Abbreviations Used
aHR $=$ adjusted hazard ratio
$\mathrm{BMI}=$ body mass index
$\mathrm{CHD}=$ coronary heart disease
$\mathrm{CI}=$ confidence interval
$\mathrm{EAU}=$ European Association of Urology
$\mathrm{KSD}=$ kidney stone disease
$\mathrm{NOS}=$ Newcastle/Ottawa Scale
$\mathrm{NR}=$ not recorded
PRISMA $=$ Preferred Reporting Items for Systematic
$\quad$ Reviews and Meta-analyses

